\title{
Hybridization of the genetic algorithm with the apparatus of fuzzy sets
}

\section{Skakalina Elena}

National University "Yuri Kondratyuk Poltava Politechnics"

Pershotravnevyi ave. 24, Poltava, UA-36011, Ukraine

wboss@i.ua

\begin{abstract}
Evolutionary algorithms are one of the effective methods for solving problems with high computational complexity, large dimension and search space. The use of evolutionary algorithms makes the question of choosing their settings and parameters relevant. The solution to this issue is difficult and significantly affects the quality of the resulting model. The paper considers the issue of determining the dimension of the current population during the operation of the genetic algorithm using the mathematical apparatus of fuzzy sets with a pentary membership scale. The proposed hybrid genetic algorithm has been tested on sets of test functions. A comparative analysis of the classical and hybrid genetic algorithms for the accuracy of solving the optimization problem of the general plan of logistics transportation is carried out.
\end{abstract}

Keywords: genetic algorithms, apparatus of fuzzy sets, optimization, logistics.

\section{INTRODUCTION AND PROBLEM STATEMENT}

The vast majority of optimization tasks require a huge investment of time and computing resources. This is due to the need to sort out a huge number of different solutions. Moreover, by their computational complexity, such problems belong to the class of so-called NP-complete problems, that is, problems for which there is no deterministic polynomial algorithm. Therefore, to ensure that the best solution (global extremum) is found in such problems, it is necessary to perform exhaustive search, which in reality is not possible due to their large dimension [1-3]. Therefore, in practice, various metaheuristic algorithms are developed to solve such problems, which allow finding close to optimal (quasi-optimal) solutions. One of the approaches that can successfully solve the problem of increasing the efficiency and quality of solving complex optimization problems of large dimension is the integration of various scientific methods specific to such areas of computational intelligence as bio-inspired algorithms, fuzzy calculations, artificial neural networks [4-5]. Genetic algorithms (GA) as representatives of the metaheuristics group have become an important tool for solving optimization problems in various fields [6].

\section{PROBLEM SOLUTION AND RESULTS}

Cost optimization is one of the most important tasks of transport logistics, the solution of which allows, under the existing restrictions on all types of resources used, to increase profits up to $25 \%$. For logistics companies, this level of cost minimization can be achieved by constructing effective route plans for vehicles (V). A particularly significant effect of cost reduction can be achieved on large logistics networks. This circumstance forces researchers to search for efficient algorithms for solving the routing problem of vehicles that generate less costly routes and schedules. Heuristics (including GA) allow in some cases to generate solutions that increase the temporary solvency of the solution by almost two times.

At the initial stage $(n=0)$ of the classical genetic algorithm, an initial population of chromosomes is randomly generated, each of which is a sequence of genes encoding an alternative solution (for example, a chromosome may encode a variant of freight transport by a specific vehicle along a specific route). In this case, each gene may carry the values of the corresponding type of $\mathrm{V}$ and the length of the route. Then begins a cycle, at each iteration of which to the current population is consistently applied: the reproduction operator, randomly selects chromosomes for crossing, in proportion to their fitness function (determined by the values of the target function of the respective pairs - V \& route); a crossover operator that mimics the generation of offspring chromosomes by borrowing separate sections of the parent's genetic code (the formation of new matching $\mathrm{V}$ pairs \& route that inherited different $\mathrm{V}$ types and routes from different previously selected old pairs); a random mutation operator, with a given (small) probability, changes the chromosome in a random place at random; and, finally, the recombination operator that determines the chromosomes that will enter the next population (selects the most appropriate for the future evolution of a pair of $\mathrm{V} \&$ route according to the value of their target function). The monetary value of the entire transport plan is used as the target function. The cycle continues until the maximum number of iterations $n$ is reached or a satisfactory solution is obtained. The scheme of traditional GA:

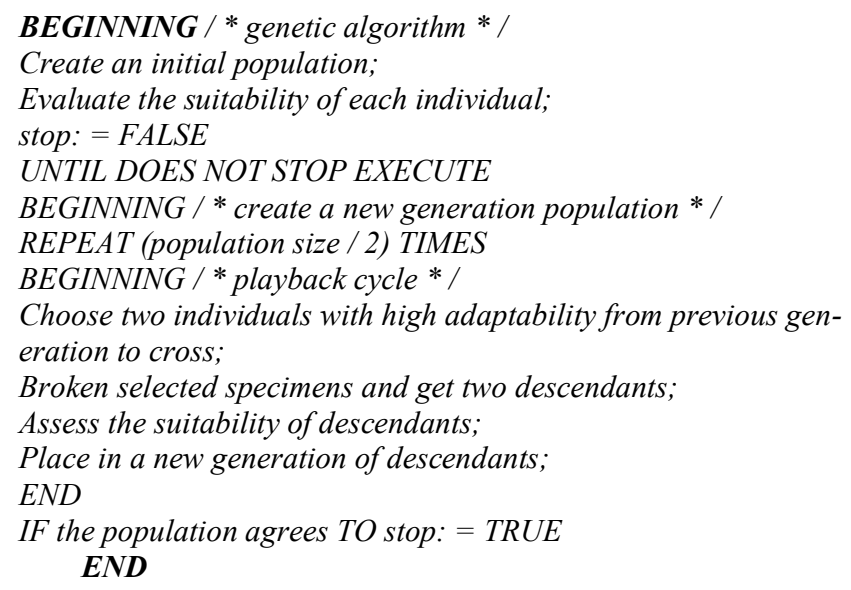

In a hybrid GA (HGA), after creating the initial population and calculating the value of the fitness function for each pair of $\mathrm{V} \&$ route, the apparatus of the theory of fuzzy sets (TFS) starts up [7]. We define a lot of linguistic variables "Value of fitness - function" as "Very Bad", "Bad", "Fair", "Good", "Very Good". Those pairs of V \& route (chromosomes), the values of the fitness-functions of which fall into the FS "Very Bad", are 
excluded from further processing. Thus, the dimension of the current population is reduced and the convergence time of the HGA is reduced, which is of relevance for problems of large dimension.

Hybrid GA Scheme:

BEGINNING / * genetic algorithm * /

Create an initial population;

Evaluate the suitability of each individual;

Formation of 5 fuzzy sets

"VB-very bad", "B-bad", "S-satisfactory", "G-good", "VG-very good" individuals depending on the suitability value / *FST * /

Reducing the dimension of the initial population by removing from it the fuzzy set " $V B$ "

stop: $=$ FALSE

UNTIL NOT STOP EXECUTE

BEGINNING / * create a new generation population * /

REPEAT (population size / 2) TIMES

BEGINNING / * playback cycle * /

Choose two individuals with high adaptability from previous gen-eration to cross;

Broken selected specimens and get two descendants;

Assess the suitability of descendants;

Formation of 5 term sets "VD", " $B$ ", "S", "G", "VG" individuals

depending on the value of the fitness function

* FST */

Reducing the dimension of the initial population by removing from it the term set "VB"

END

IF the population agrees TO stop: $=$ TRUE

END

The following HGA characteristics were used in the work: crossover type - single-point, parameter coding - real, which gives additional advantages in terms of HGA operation speed; the FS apparatus is used as a strategy for the formation of a new generation. The results of the first stage of experiments on test functions showed that the HGA showed the best results in terms of convergence rate and the probability of reaching an absolute optimum in comparison with classical GA (Table 1).

Table 1. Results of the first stage of testing

\begin{tabular}{|l|l|l|l|}
\hline Type GA & \multicolumn{3}{|c|}{ Testing function } \\
\hline & De Jong & Rosenbrock & Rastrigin \\
\hline CGA & 0.89 & 0.86 & 0.84 \\
\hline HGA & 0.92 & 0.90 & 0.88 \\
\hline
\end{tabular}

The results of the second experiment confirmed the results of the first. The value of the total cost of the entire transportation plan obtained using HGA showed more optimal results compared to CGA (Table 2).

Table 2. Value of the cost of the general transportation plan

\begin{tabular}{|l|l|l|}
\hline $\begin{array}{l}\text { Domain } \\
\text { Object }\end{array}$ & CGA (\$) & HGA (\$) \\
\hline DO\#1 & 21598.70 & 16124.61 \\
\hline DO\#2 & 83305.60 & 65984.84 \\
\hline DO\#3 & 58179.20 & 47630.10 \\
\hline DO\#4 & 112384.67 & 94781.93 \\
\hline DO\#5 & 25384.98 & 12021.75 \\
\hline DO\#6 & 97359.50 & 82840.36 \\
\hline DO\#7 & 25292.50 & 16977.14 \\
\hline DO\#8 & 11091.83 & 7308.28 \\
\hline DO\#9 & 273595.78 & 223387.96 \\
\hline DO\#10 & 308625.32 & 317943.95 \\
\hline DO\#11 & 233056.21 & 215091.83 \\
\hline
\end{tabular}

The proposed HGA is programmatically implemented on the .NET Framework and the MS Visual Studio development shell, the programming language is $\mathrm{C}$ \#. Experiments were conducted to evaluate the capabilities of the author's HGA. The first stage of the HGA testing was carried out on the test functions of De Jong, Rozenbrock, Rastrigin [8-10]. The second stage of testing was carried out on real data obtained at the facilities of the following subject areas - the agricultural industry, the oil and gas industry, the logistics area in the process activity.

\section{CONCLUSIONS}

Evolutionary algorithms in general and genetic algorithms in particular have an advantage in solving optimization problems. This advantage is their ability to simultaneously operate with many solutions - the population, which allows us to reach a global extremum without getting stuck in local ones. Moreover, information about each individual in the population is encoded on the chromosome (genotype), obtaining the optimal solution (phenotype) is obtained after the implementation of the evolution process (selection, crossing, mutation) after decoding. The HGA proposed in the work allows the adaptive process of regulating the sizes of the initial and current populations using TFS. Dividing the current populations into five term sets makes it possible to implement parallel execution of HGAs (the so-called spatial methods for finding the extremum), which are generally more efficient than sequential (temporary) ones. In addition, in many subject areas of GA application in the formation of genes and chromosome structure, it is irrational to use binary coding and mutation, which is used in binary coding. This circumstance provides an additional advantage in increasing the temporary solvency of the HGA. Being randomly directed search methods on a variety of solutions, GAs are effectively used to solve NP-complete problems in decision support systems in complex organizational and technical systems.

\section{REFERENCES}

[1] Cohoon J.P., Karro J., Lienig J. Evolutionary algorithms for the physical design of VLSI circuit. In: Advances in Evolutionary Computing: Theory and Applications. A. Ghosh, S. Tsutsui (Eds.). Springer Verlag, London, 2003, pp. 683-712.

[2] Shervani N. Algorithms for VLSI physical design automation. Kluwer Acad. Publ., Dordrecht, 1995. 538 p.

[3] Alpert Ch.J., Dinesh P., Mehta D.P., Sapatnekar S.S. Handbook of algorithms for physical design automation. CRC Press, NY, USA, 2009.

[4] Ershov N.M. Non-uniform cellular genetic algorithms // Computer Research and Modeling, 2015, vol. 7, no. 3, pp. 775-780.

[5] Whitfield-Gabrieli S and Nieto-Castanon A, "Conn: a functional connectivity toolbox for correlated and anticorrelated brain networks," Brain Connect., vol. 2, no. 3, pp. 125-141, 2012. https://doi.org/10. 1089/brain.2012.0073 PMID: 22642651.

[6] Kazakovtsev, L.A. An Approach to the Multi-facilityWeber Problem with Special Metrics /L.A.Kazakovtsev, P.S.Stanimirovic // European Modelling Symposium (EMS), 20-22 Nov. 2013.-Manchester:UkSim.2013.-P. 119-124. DOI:10.1109/EMS.2013.21.

[7] Skakalina, E. (2018), «Development of Methodological Foundations of Logistical Intellectual Control of Complex Systems Based on Hybrid Heuristic Algorithms» / International Journal of Engineering \& Technology.- 2018.- Vol. 7, No (4.8). - P.534-538. DOI: $\underline{10.14419 / \text { ijet.v7i4.8.27301 }}$

[8] De Jong K.A. Evolutionary computation a unified approach // A Bradford book. Cambridge: MA, USA. 256 p.

[9] Rosenbrock H.H., An automatic method for finding the greatest or least value of a function. - The Computer Journal 3, 1960. pp. 175-184.

[10] Rastrigin L. A., Systems of Extremal Control - Nauka, Moscow, 1974. 